\title{
Doubling Hepatitis C Virus Screening in Primary Care Using Advanced Electronic Health Record Tools-A Non-Randomized Controlled Trial
}

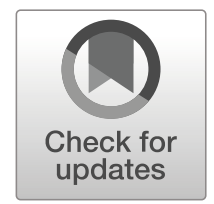

\author{
Leila Hojat, MD ${ }^{1,2}$, Ann Avery, MD², Peter J. Greco, MD², and David C. Kaelber, MD, \\ $\mathrm{PhD}, \mathrm{MPH}^{2,3,4}$
}

\begin{abstract}
'Division of Infectious Diseases and HIV Medicine, University Hospitals Cleveland Medical Center, Case Western Reserve University, Cleveland, OH, USA; ${ }^{2}$ Department of Medicine, The MetroHealth System, Case Western Reserve University, Cleveland, OH, USA; ${ }^{3} \mathrm{Center}$ for Clinical Informatics Research and Education, The MetroHealth System, Case Western Reserve University, Cleveland, OH, USA; ${ }^{4}$ Department of Pediatrics and Department of Population and Quantitative Health Sciences, Case Western Reserve University, Cleveland, OH, USA.
\end{abstract}

BACKGROUND: Hepatitis C virus (HCV) infection is a major public health burden, affecting over 4 million people. The Centers for Disease Control and Prevention and the US Preventive Services Task Force guidelines recommend screening everyone born between 1945 and 1965, but screening rates remain low.

OBJECTIVE: To determine whether bulk ordering and electronic messaging to patients improves guidelinebased HCV screening rates.

DESIGN: A non-randomized controlled trial of 1024 adults from November 2016 to March 2017.

PARTICIPANTS: Patients due for HCV screening with at least one primary care office visit in one of three primary care clinics and enrolled in the healthcare system's tethered personal health record (tPHR).

INTERVENTIONS: Control patients received normal care for HCV screening, consisting of passive HCV reminders to providers during face-to-face visits and passive $\mathrm{HCV}$ screening notification through the patient's tPHR. Intervention patients received normal care and also had HCV antibody tests ordered for them and customized messages sent through their tPHR inviting them to go directly to the lab for HCV screening over a 12 -week period.

MAIN MEASURES: Percentage/number of patients receiving HCVantibody tests during the intervention period. Percentage/number of intervention group patients receiving HCV screening with other blood work.

KEY RESULTS: In the intervention group, 33\% (168 of 514) completed HCV testing, compared with 19\% (97 of 510) of controls (OR 1.7, 95\% CI 1.2-2.1). Bulk lab ordering appeared to have a large impact while bulk messaging appeared to have a less significant role.

CONCLUSIONS: Leveraging population analytics and bulk ordering in an electronic health record with bulk messaging to a tPHR directly engages patients in blood screening tests and can significantly improve completion. This methodology has a broad range of applications including many recommended screening or disease-specific testing. This bulk ordering and direct-to-patient messaging approach improves patient screening while decreasing provider/staff work.

Received January 17, 2019

Revised July 26, 2019

Accepted October 11, 2019

Published online December 2, 2019
TRIAL REGISTRATION: MetroHealth IRB16-00776 (ClinicalTrials.gov)

KEY WORDS: hepatitis C virus; screening; electronic health records; personal health records; population health management.

J Gen Intern Med 35(2):498-504

DOI: $10.1007 / \mathrm{s} 11606-019-05536-Z$

(C) Society of General Internal Medicine 2019

\section{INTRODUCTION}

Hepatitis $\mathrm{C}$ virus $(\mathrm{HCV})$ infection represents a major public health burden in the USA. Based on 2013-2016 NHANES data, the estimated prevalence of positive anti-HCV antibodies in the USA is $1.7 \%$, representing approximately 4.1 million individuals. ${ }^{1}$ Approximately $80 \%$ of these positive antibody results represent chronic HCV infection, which is a major source of morbidity and has higher rates of mortality than $\mathrm{HIV}^{2,3}$ The largest proportion of chronically infected individuals are adults born between 1945 and 1965; although only encompassing $27 \%$ of the US population, they account for roughly three-quarters of all HCV infections. ${ }^{4}$ Thus, the Centers for Disease Control and Prevention (CDC) in 2012 and the United States Preventive Services Task Force (USPSTF) in 2013 have recommended universal one-time HCV antibody testing for all adults born within this time period, regardless of other risk factors. ${ }^{5}$

Despite these guidelines, $\mathrm{HCV}$ testing and identification remain poor. Only $15 \%$ of those infected are aware of their status. $^{6}$ Among US Veterans, only $51 \%$ of patients seen regularly among the birth cohort born between 1945 and 1965 had received HCV testing. ${ }^{7}$ Prior studies have investigated approaches to improve compliance with national screening guidelines regarding viral infections. Studies typically rely on trying to improve screening at face-to-face encounters, either primary care, emergency departments, inpatient settings, or even prisons. ${ }^{8-12}$ These studies show that $\mathrm{HCV}$ screening can be improved during face-to-face encounters, but also that these improvements can be difficult to sustain over time because they require continued staff engagement 
over time and/or significant continued resource investment. Further, these studies do not address patients that do not present for face-to-face encounters.

Here, we present a study to evaluate the effectiveness of advanced electronic health record (EHR) population health tools, bulk ordering, and active tethered personal health record (tPHR) messaging among patients due for HCV screening, based on their birth year. A tPHR is a website or mobile application that a patient uses to interact with information and staff in a specific EHR. Common tPHR functions include reviewing test results, messaging to and from patients, providers, and other healthcare system staff, paying healthcare bills, renewing medications, and scheduling appointments. ${ }^{13}$

\section{METHODS}

We selected patients seen for primary care in our healthcare system (The MetroHealth System, Cleveland $\mathrm{OH})$ in three large primary care clinics and due for $\mathrm{HCV}$ testing based on their birth year, identified using population health data tools embedded within our EHR (EpicCare Systems, Verona, WI). Inclusion criteria included the following: (1) being in the birth cohort for HCV screening (born between 1945 and 1965), (2) being seen in the prior 6 months in one of the primary care study sites (so that they had at least one face-to-face opportunity for HCV screening was missed), (3) having an activated tPHR account, (4) not having an HCV antibody test on file in our healthcare system, and (5) being marked as alive in our EHR at the time of study enrollment. Exclusion criteria included the following: (1) any documented HCV viral load level based on laboratory testing in our EHR, (2) any encounter within our liver clinic (which follows the majority of HCV-positive patients), and (3) any diagnosis of "chronic HCV," "cirrhosis secondary to HCV," or "hepatitis C antibody positive test" based on ICD codes in the patient's encounter diagnoses, problem list, or past medical history diagnoses in our EHR (Fig. 1). Each clinic had its own control and intervention groups, with a total of 514 patients in the intervention group and 510 patients in the control group across all three clinics. Patients were selected to be in the control or intervention group based on the first letter of the patient's last name. No methods changed after study initiation.]->

Electronic messages through the EHR were sent to all primary care providers in the three study clinics (about 75 providers) notifying them that some of their patients would be enrolled in this population health program. Primary care providers were blinded to which of their patients were in each group. Control patients received normal care for HCV screening, consisting of passive HCV screening health maintenance reminders to providers during face-to-face visits and passive HCV screening notification through the patient's tPHR. For intervention patients, using our EHR's population health tools, bulk orders where placed for HCV antibody testing. Additionally, bulk messages were sent through their tPHR inviting them to go directly to the lab for HCV screening over a 12week period, in additional to usual care (Fig. 2). The bulk messages were customized messages automatically generated by standard population health tools in our EHR and sent to the patients' tPHR (Fig. 3) notifying them that they were due for $\mathrm{HCV}$ testing and providing a brief explanation of $\mathrm{HCV}$ and why HCV testing was being recommended. When the message was sent to the tPHR, a "tickler" message to check the tPHR was sent to the patient's email address on file. The message instructed patients to go to the nearest lab facility within the healthcare system, all of which were listed at the bottom of the message. Message wording was written at an 8th grade reading level of comprehension, measured via an online readability calculator (https://readable.io).] $\rightarrow$ ]->

HCV completion data was collected 1 week, 2 weeks, 4 weeks, 8 weeks, and 12 weeks post-intervention (November 2016-March 2017). The automatically generated message was resent after 4 weeks to all individuals in the intervention group who had not already received testing to date to determine if repeat messaging effected the testing rate. Additional new encounters and/or additional labs were also noted to determine if HCV labs were drawn separately or in conjunction with other ordered laboratory tests.

All positive HCV antibody tests automatically had HCV RNA viral load testing (standard reflex testing within our laboratory) to determine if the patient had cleared the HCV spontaneously or if they remained chronically infected. Positive HCV test results were routed via the EHR to a prespecified infectious disease specialist involved in HCV care. This specialist followed-up with the patient's primary care provider and/or the patient themselves (depending on primary care provider preference) to discuss the implications of the positive test results and help obtain follow-up evaluation and treatment, if needed.

The primary outcome included total HCV screenings documented as completed in our EHR in the control and intervention groups at the end of the study period across all three study sites. Secondary outcomes, also assessed at the end of the study period, included (1) average time for screening after messaging, (2) HCV testing with and without concurrent other blood tests, (3) effect of repeat messaging and age and gender of patient on HCV screening in the intervention group, and (4) any patient and provider comments related to $\mathrm{HCV}$ messaging. All statistical analysis was performed using medcalc.org statistical and Excel software. All analyses were developed a priori with a $p$ value of $<0.05$ considered statistically significant. Sample size was based on our prior experience of a $25 \%$ effectiveness of patient messaging. ${ }^{14}$ This study was powered to detect a difference of at least $10 \%$ in overall screening between the control and intervention groups. This study was approved by the MetroHealth System Institutional Review Board. 


\section{1,024 patients with:}

1) Born between 1945-1965

2) Seen in one of the three primary care sites of interest in the last 6 months

3) Enrolled in personal health record (MyChart) (currently)

4) Do not have an HCV antibody on file (ever)

5) Are currently marked in the EHR as alive

1) HCV viral load (ever)

2) Liver clinic visit (ever)

3) Diagnosis of "chronic HCV", "cirrhosis secondary to HCV", or "hepatitis C antibody positive test" on their problem list or in their past medical history diagnoses

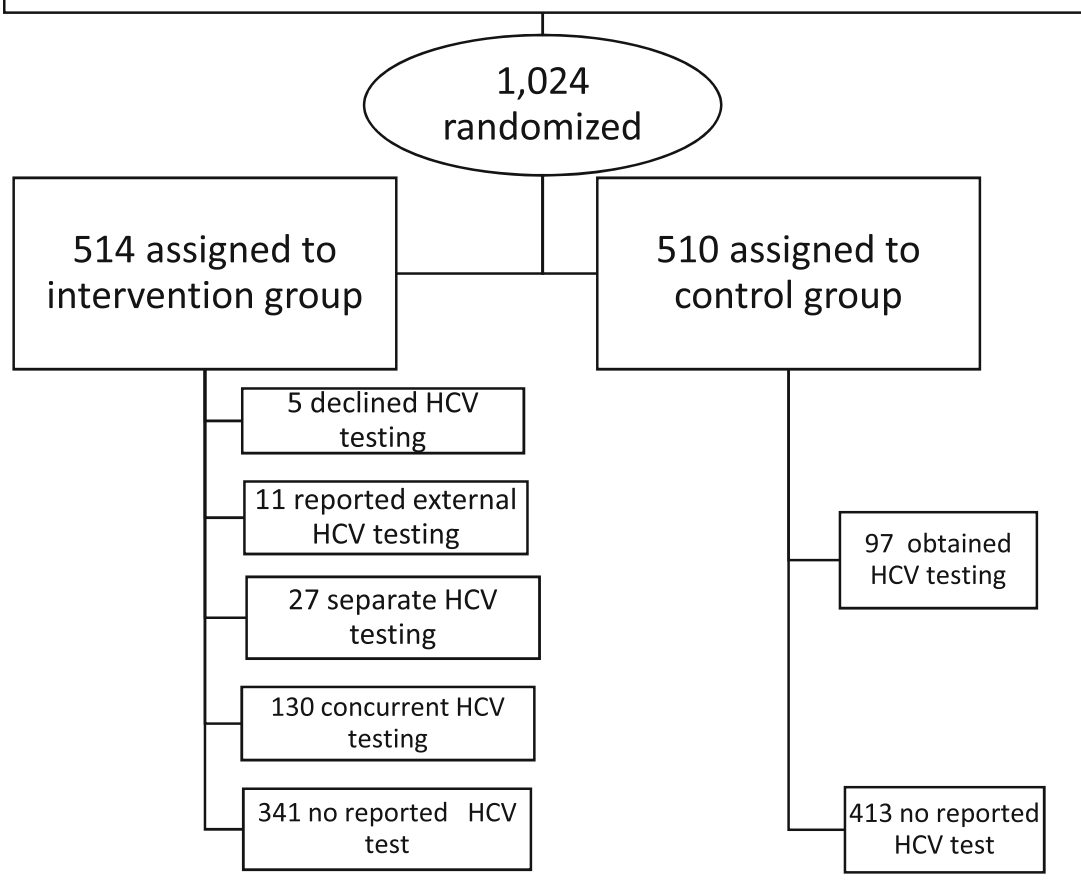

Figure 1 Cohort and subsamples population diagram. HCV hepatitis C virus.

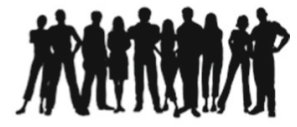

510 people control group

\section{And without:}




\title{
Dear PATIENT PREFERRED FIRST NAME,
}

\begin{abstract}
Based on our records, it looks like you are due for hepatitis $C$ testing. National guidelines recommend testing everyone born between 1945 and 1965. Hepatitis $\mathrm{C}$ is a virus which causes liver disease. Many people do not know they are infected with hepatitis $C$, so it is important to test people at risk. Effective treatment for hepatitis $C$ is now available.
\end{abstract}

For more information about hepatitis $\mathrm{C}$ visit this link: http://www.cdc.gov/hepatitis/hcv/cfaq.htm.

\author{
be automatically done too. \\ Thank you. \\ Leila Hojat, MD \\ MetroHealth Population Health Management \\ Walk-In Lab Facilities: \\ MetroHealth Medical Center \\ 2500 MetroHealth Drive \\ Cleveland, $\mathrm{OH} 44109$ \\ Labs: 216-778-5185 \\ Lab Hours \\ Monday - Friday \\ 7:00 a.m. - 5:45 p.m
}

Please go to the nearest MetroHealth lab to get your blood drawn. See below for hours and locations. You do not need an appointment. If you have other blood work done, this test will

Figure 3 Sample of tethered personal health record message sent to patients (only one MetroHealth laboratory location listed, although the actual letter had 6 locations).

\section{RESULTS}

\section{Primary Outcomes}

A total of 514 patients were selected for the intervention group and 510 were selected for the control group (Table 1). There was no difference in gender between the two groups (both $42 \%$ male) and patients in the intervention group were on average 1 year younger ( 59 versus 60 ) than the control group. In the intervention group, $168(33 \%)$ participants completed testing compared with $97(19 \%)$ in the control group ( $p$ value $<0.0002)$. This corresponded to an odds ratio of 1.7 (95\% confidence interval (CI) 1.2-2.1). Among the 168 individuals who completed testing in the intervention group, $130(75 \%)$ had concurrent lab testing. 27 individuals (16\%) had a separate HCV antibody level drawn without concurrent labs. During the intervention period, 11 patients were identified that had HCV testing outside of our healthcare system, based on messages from the patient sent to us through the tPHR indicating this. We were sometimes able to validate external test

Table 1 Study Group Characteristics

\begin{tabular}{llll}
\hline \hline & $\begin{array}{l}\text { Control group } \\
(\boldsymbol{n}=\mathbf{5 1 0})\end{array}$ & $\begin{array}{l}\text { Intervention group } \\
(\boldsymbol{n}=\mathbf{5 1 4})\end{array}$ & $\boldsymbol{p}$ value \\
\hline Patient total & 510 & 514 & \\
1st site & 81 & 83 & \\
2nd site & 345 & 346 & \\
3rd site & 84 & 85 & 0.03 \\
Average age & 59 years & 60 years & 1 \\
Male & $42 \%$ & $42 \%$ & $<$ \\
Completed HCV & $19 \%(97)$ & $33 \%(168)$ & 0.0002 \\
testing & & & \\
\hline
\end{tabular}

HCV hepatitis $C$ virus completion through health information exchange and sometimes not, based on that other healthcare systems not always being able to exchange data with us electronically. Five patients declined testing, and their charts were updated to reflect this. Patient with self-reported or confirmed HCV testing outside of our healthcare system were counted as having "completed" testing. Those declining testing were not considered to have completed testing, although their charts were updated with this information to indicate a prior HCV screening attempt.

\section{Secondary Outcomes}

Age and gender did not have a statistically significant effect on test completion $(p>0.05)$. The average time to complete testing in the intervention group was 7.3 weeks (SD 4 weeks). Repeat messaging did not appear to have an impact on test completion. In terms of patients' comments, eighteen patients (3.5\%) replied to their tPHR message. Most (9) expressed thanks or acknowledgment at having received the message. Four self-reported negative $\mathrm{HCV}$ tests at another facility, not accessible through electronic health information exchange. One patient had moved to another state but still expressed thanks for the reminder. One patient asked for the HCV antibody order to be sent to a different lab which would charge their insurance company less. Two patients contacted their primary care providers to determine if the lab test had been a mistake, given that the lab was ordered by an unknown provider. One patient expressed concern and frustration that their insurance might unnecessarily be charged. Providers provided feedback with EHR messages or other personal communications with the authors. In one case, a 
provider indicated the patient's HCV testing status as declined, but only after the lab was already ordered. A small number of providers $(<5)$ expressed concern about screening notifications being sent to their patients without their direct involvement. Another small group of providers $(<5)$ had concerns about answering questions regarding an intervention in which they were not directly involved in.

\section{DISCUSSION}

Our strategy resulted in an almost doubling (19 to 33\%) of compliance with preventive care guidelines involving blood work for HCV screening. Population health programs to improve screening rates are important clinically because for diseases with long asymptomatic periods, such as HCV, increasing screening can lead to significant decreases in morbidity and mortality. Additionally, as healthcare moves from fee-for-service to pay-for-performance, compliance with screening tests are routinely incorporated into these pay-forperformance programs. Compliance with screening programs is also important because of their general cost-effectiveness secondary to earlier disease detection. Our novel approach to improving screening rates used advanced EHR integrated population health tools coupled with a tPHR. This integrated technology driven approach utilized cohort identification, bulk ordering, and bulk direct messaging to patients outside of faceto-face visits. Our increase is despite the study population being routinely followed in our healthcare system with passive EHR health maintenance reminders at face-to-face visits and passive tPHR reminders.

The OR that we found of 1.7 is consistent with the impact of other EHR tools to improve adherence to preventive care measures. A meta-analysis of computer-based reminder systems performed by Shea and colleagues identified sixteen randomized, controlled trials showing that specific preventive care practices including immunizations, cardiovascular care, cancer screening, and smoking cessation improved with an adjusted OR of 1.77 for all areas combined. ${ }^{15}$ Despite these studies, a 2012 Cochrane analysis only found moderate quality evidence indicating that computer-generated reminders only moderately (median 7\%) improved care processes. ${ }^{16}$ One of the primary issues might be that preventive care reminder tools are typically directed at providers/staff and not the patient themselves. Although direct-to-patient messaging has the potential to increase duplicative testing, as patients become more engaged in their care through more robust tPHRs and health information exchanges improve, actual duplicative testing will be low. We are not aware of any actual duplicative testing that occurred through this project. Directing appropriate patient care reminders to patients themselves, in those cases where providers/staff do not need to be intermediaries, increases the reminders' effectiveness while at the same time increasing provider/staff efficiency and decreasing provider/staff workload.
Prior interventions targeting HCV antibody testing have involved provider/staff training or focus on outreach to highrisk patients. ${ }^{17}$ While provider-based interventions improved testing rates and linkage to care, the resources required to perform these types of interventions in terms of provider and staff time are high. Sidlow and Msaouel designed an EHRbased targeted intervention involving a decision support module that triggered an automatic test order for patients who were eligible at the time of visit. ${ }^{18}$ This intervention increased screening rates among patient seen by $254 \%$ (from 11 to $49 \%$ ), but relied on face-to-face visits and providers signing orders. The $74 \%$ increase in screening measured in our intervention group is in addition to normal care reminders. In our healthcare system and in meta-studies, simple reminders, like those in our normal care control group, have been shown to improve screening rates by 7 to $77 \% .^{15,16}$

We previously employed direct messaging to patients to improve adolescent immunization rates. ${ }^{14}$ In that study, the number needed to message (NNM) (i.e., the number of people needed to message to have one additional person receive the intervention) was about four, generally consistent with the NNM in this study of about six. Our systematic approach to $\mathrm{HCV}$ screening also addresses many of the barriers identified for viral disease screening including insufficient time, lack of knowledge or training, lack of patient acceptance, competing priorities, and inadequate reimbursement. ${ }^{19}$

Our study linked two related, yet separate interventions - bulk ordering and bulk messaging. The HCV antibody order was bulk ordered such that any time after the order was signed and the patient presented to one of our healthcare system labs for any blood work, the HCV testing would be completed. The bulk messaging to patients in their tPHR served as an active reminder to them that the HCV antibody order has been placed (as well as background into what $\mathrm{HCV}$ was and why screening was important). $75 \%$ of the $\mathrm{HCV}$ testing occurred in conjunction with other blood work. In these patients, the patient may not have realized/understood that the HCV test was being conducted with the other testing being done, although all patients received notification in their tPHR about the HCV antibody order. This potentially brings up autonomy issues related to patient's informed consent.

Our enhanced testing (at a cost of $\sim \$ 50$ per HCV test) lead to new chronic HCV diagnoses in two asymptomatic patients. Given the estimated lifetime cost savings of early diagnosis of an individual with $\mathrm{HCV}$ infection to be at $\$ 64,490$, this translates to a savings of nearly $\$ 130,000 .{ }^{20}$ With the introduction of additional direct-acting antiviral regimens, the cost of treatment has decreased, making early diagnosis (prior to symptom onset) and treatment a cost-effective approach. ${ }^{21}$ Furthermore, early diagnosis and treatment reduces symptoms, improves quality of life, and reduces all-cause mortality; thus, the American Association for the Study of Liver Diseases-Infectious Disease Society of America current guidelines recommend treatment of HCV infection for all individuals except those 
with short life expectancies that cannot be expected to improve with treatment of $\mathrm{HCV}$ or transplantation (class 1, level A). ${ }^{22}$

The implications of our findings are significant, as this type of strategy could easily be broadened to large populations with an integrated EHR, imbedded population health tools, and a tPHR. Additionally, this approach could be applied to other recommended universal screening tests such as HIV and lipid testing or disease-specific labs such as renal function tests and glycosylated hemoglobin in diabetic patients. Keys to this type of approach include the following: (1) administrative issues (cost, insurance coverage, and testing capacity), (2) communication approach with providers (especially primary care providers) and patients, and (3) follow-up approach for abnormal tests.

Our study has limitations. First of all, it was difficult to determine the separate effects of bulk ordering versus bulk messaging. Individuals could have had their HCV antibody level drawn along with other labs and be unaware that the $\mathrm{HCV}$ antibody level was concurrently being checked. Some individuals were probably aware that they were due for this testing (either seeing an alert in their tPHR or reading the message sent as part of this intervention) but waited to go to the lab because they were expecting additional lab work in the near future (e.g., they had an upcoming appointment). Secondly, related to generalizability, this study was performed within an integrated health delivery system with a single instance of an EHR with fully integrated, advanced population health and tPHR functionality, all from the same vendor. However, the functionality presented here (all commercially available — no additional cost within our EHR) could be implemented in any healthcare system today $(\sim 1$ hour effort for initial query, bulk ordering, and bulk messaging; $\sim 15$ minutes for subsequent queries, ordering, and messaging). Although these interventions were limited to patients with an activated tPHR account, patient messaging through the same bulk ordering and bulk messaging tools could be used to print and mail bulk letters, although typically at an additional cost of $\sim$ $\$ 2.50$ per letter for printing, stuffing, and mailing a letter. Finally, this intervention was only conducted in patients born between 1945 and 1965, so it is unproven if this approach would be more or less effective in different patient age groups or for different types of blood tests. However, at least in terms of different age groups, younger patients are typically thought to be more technology engaged and so this approach may be more effective in younger patients.

\section{CONCLUSION}

This study describes a novel, generalizable population health approach for improved screening, leveraging advanced EHRbased population health tools coupled with tPHRs. These tools and techniques can be used to automate screening programs for bloodwork (and other testing) and demonstrates that EHRbased population health tools can be used to screen for $\mathrm{HCV}$ infection in accordance with national guidelines, independent of a face-to-face patient-provider encounter. This direct to patient approach leads to higher screening rates while at the same time decreasing provider/staff work. EHR-based population health tools such as these will be essential for healthcare systems to adopt as pay-for-performance models of care become increasingly important.

Corresponding Author: David C. Kaelber, $M D, P h D, M P H$; Shaker Heights, OH, USA

\section{Compliance with Ethical Standards:}

Conflict of Interest: The authors declare that they do not have a conflict of interest.

\section{REFERENCES}

1. Hofmeister MG, Rosenthal EM, Barker LK, et al. Estimating prevalence of hepatitis C virus infection in the United States, 2013-2016. Hepatology 2019;69:1020-31.

2. Klevens RM, Hu DJ, Jiles R, Holmberg SD. Evolving epidemiology of hepatitis C virus in the United States. Clin Infect Dis 2012;55 Suppl 1:S3-9.

3. Ly KN, Xing J, Klevens RM, Jiles RB, Ward JW, Holmberg SD. The increasing burden of mortality from viral hepatitis in the United States between 1999 and 2007. Ann Intern Med 2012;156:271-8.

4. Smith BD, Morgan RL, Beckett GA, et al. Recommendations for the identification of chronic hepatitis $\mathrm{C}$ virus infection among persons born during 1945-1965. MMWR Recomm Rep 2012;61:1-32.

5. Moyer VA. Screening for hepatitis $C$ virus infection in adults: U.S. Preventive Services Task Force Recommendation Statement. Annals of Internal Medicine 2013; 159:349-57.

6. Easterbrook P, Johnson C, Figueroa C, Baggaley R. HIV and hepatitis testing: global progress, challenges, and future directions. AIDS Rev 2016;18:3-14.

7. Sarkar S, Esserman DA, Skanderson M, Levin FL, Justice AC, Lim JK. Disparities in hepatitis C testing in U.S. veterans born 1945-1965. J Hepatol 2016;65:259-65.

8. Rayment M, Thornton A, Mandalia S, et al. HIV testing in nontraditional settings - the HINTS Study: a multi-centre observational study of feasibility and acceptability. PLoS ONE 2012;7:e39530.

9. Rumble C, Pevalin DJ, O'Moore E. Routine testing for blood-borne viruses in prisons: a systematic review. Eur J Public Health 2015;25: 1078-88.

10. O'Connell S, Lillis D, Cotter A, et al. Opt-out panel testing for HIV, hepatitis B and hepatitis $\mathrm{C}$ in an urban emergency department: a pilot study. PLoS One 2016;11:e0150546.

11. Turner BJ, Taylor BS, Hanson JT, et al. Implementing hospital-based baby boomer hepatitis $\mathrm{C}$ virus screening and linkage to care: strategies, results, and costs. J Hosp Med 2015;10:510-6.

12. Rayment M, Rae C, Ghooloo F, et al. Routine HIV testing in the emergency department: tough lessons in sustainability. HIV Med 2013;14 Suppl 3:6-9.

13. Roehrs A, da Costa CA, Righi RdR, de Oliveira KSF. Personal health records: a systematic literature review. J Med Internet Res 2017;19:e13.

14. Bar-Shain DS, Stager MM, Runkle AP, Leon JB, Kaelber DC. Direct messaging to parents/guardians to improve adolescent immunizations. J Adolesc Health 2015;56:S21-6.

15. Shea S, DuMouchel W, Bahamonde L. A meta-analysis of 16 randomized controlled trials to evaluate computer-based clinical reminder systems for preventive care in the ambulatory setting. J Am Med Inform Assoc 1996;3:399-409.

16. Arditi C, Rege-Walther M, Wyatt JC, Durieux P, Burnand B. Computergenerated reminders delivered on paper to healthcare professionals; effects on professional practice and health care outcomes. Cochrane Database Syst Rev 2012;12:Cd001175.

17. Aspinall EJ, Doyle JS, Corson S, et al. Targeted hepatitis C antibody testing interventions: a systematic review and meta-analysis. Eur J Epidemiol 2015;30:115-29. 
18. Sidlow R, Msaouel P. Improving hepatitis C virus screening rates in primary care: a targeted intervention using the electronic health record. $\mathrm{J}$ Healthc Qual 2015;37:319-23.

19. Burke RC, Sepkowitz KA, Bernstein KT, et al. Why don't physicians test for HIV? A review of the US literature. Aids 2007;21:1617-24.

20. El Khoury AC, Klimack WK, Wallace C, Razavi H. Economic burden of hepatitis C-associated diseases in the United States. J Viral Hepat 2012;19:153-60.

21. Panneer N, Lontok E, Branson BM, et al. HIV and hepatitis C virus infection in the United States: whom and how to test. Clin Infect Dis 2014;59:875-82.
22. HCV testing and linkage to care. Recommendations for testing, managing, and treating hepatitis C. (Accessed 10/03/2019, at http://www. hcvguidelines.org/full-report/hcv-testing-and-linkage-care.)

Publisher's Note Springer Nature remains neutral with regard to jurisdictional claims in published maps and institutional affiliations. 